\title{
DESAFIOS E CONQUISTAS DA APICULTURA NO VALE DO PARAÍBA-SP: A JORNADA DOS EMPREENDEDORES APÍCOLAS FAMILIARES ${ }^{1}$
}

\author{
ACHIEVEMENTS AND CHALLENGES OF BEEKEEPING IN THE VALLEY PARAÍBA-SP: A \\ JOURNEY OF FAMILY BEE ENTREPRENEURS \\ Luiz Eugênio Veneziani Pasin ${ }^{2}$ \\ Lídia Maria Ruv Carelli Barreto ${ }^{3}$ \\ Elisa Maria Pires Souza ${ }^{4}$ \\ Ana Paula Dib ${ }^{3}$ \\ João Carlos Nordi ${ }^{3}$
}

Resumo: Em 2007, visando aproveitar o potencial da região do Vale do Paraíba-SP, melhorar o sistema de produção e promover ações empreendedoras entre os apicultores familiares, iniciou-se a construção das ações que resultou na metodologia denominada "Ciranda do Mel". Esta possibilitou uma ação multidisciplinar que envolveu diversos atores e agentes socioprodutivos com alcance de resultados surpreendentes ao longo destes 07 anos. Esta metodologia de ações participativas se fundamenta na atuação coletiva entre os participantes e o envolvimento dos familiares, onde durante a execução do programa são realizadas visitas mensais, treinamentos; discussão das ações; aplicação de tarefas e rodízio de acompanhamento dos resultados específicos nos apiários dos apicultores participantes. A "Ciranda do Mel" com o passar do tempo incorporou novos propósitos, que permitiram ampliar o horizonte dos apicultores e seus familiares, alcançando um nível de arranjo e organização sócio produtiva, que viabilizou o alcance em outras áreas além da apicultura, como o desenvolvimento das artes, artesanato, culinária, cosméticos e produção de olerícolas. Atualmente o maior desafio dos grupos está na capacidade de se organizar para conquistar de maneira plena o acesso aos novos mercados institucionais (Programa de Aquisição de Alimentos-PAA e ao Programa Nacional de Alimentação Escolar - PNAE), pois estas novas oportunidades têm possibilitado o surgimento de novos empreendimentos associativos e a comercialização da produção ou parte dela.

Palavras-chave: Apicultura familiar. Arranjo sócio produtivo. Empreendedorismo. Mel.

Abstract: In 2007, seeking to explore the potential of the Paraiba Valley - SP, improve the system of production and to promote entrepreneurial activities among family beekeepers began the construction of the actions that resulted in a methodology called "Ciranda do Mel" (Honey Ciranda). This enabled a multidisciplinary action involving many actors and socioproductive agents with a range of amazing results over these 07 years. This methodology of participatory actions is based on collective activity among the participants and the involvement of family members, where during the program execution are performed monthly visits, trainings; discussion of the actions, implementation of tasks and rotation of the specific results monitoring on the participating beekeepers apiaries. The "Ciranda do Mel" over time has incorporated new purposes, which enabled it to expand the horizon of the beekeepers and their families, reaching a level of arrangement and socio-productive organization, which enabled the range to reach other areas than beekeeping, such as the development of arts, crafts, cooking, cosmetics and production of vegetable crops. Currently the biggest challenge lies in the ability of the groups to organize to gain full access to the new way institutional markets (Food Acquisition Program-PAA and the National School Feeding Programme - PNAE) because these new opportunities have enabled the emergence the new associative ventures and the marketing of the production or part thereof.

Key words: Family beekeeping. Socioproductives arrangement. Entrepreneurship. Honey.

1 Os autores agradecem aos parceiros institucionais que viabilizaram a elaboração dos projetos mediante ao apoio financeiro e operacional.

2 Professor da Universidade Federal de Itajubá-UNIFEI. E-mail: luiz.pasin@yahoo.com.br.

3 Professor(a) da Universidade de Taubaté-UNITAU.

4 Coordenadora do Projeto Apicultura Sustentável no município de Monteiro Lobato-SP. 


\section{INTRODUÇÃO}

Visando aproveitar o potencial apícola da região do Vale do Paraíba-SP, mediante a melhoria no sistema de produção e a própria promoção do comportamento empreendedor entre os apicultores familiares, em 2007 o Centro de Estudos Apícolas da Universidade de Taubaté iniciou a caminhada para o desenvolvimento da atividade da apicultura sustentável, onde o processo de construção das ações extensionistas resultou na metodologia denominada "Ciranda do Mel" e possibilitou uma ação multidisciplinar que envolveu diversos atores e agentes sócio produtivos com alcance de resultados surpreendentes ao longo destes 07 anos de experiências vividas e relatadas.

$\mathrm{O}$ início do processo de formação dos arranjos socioprodutivos da apicultura teve origem no ano de 2007, quando foi elaborado o projeto de desenvolvimento da apicultura familiar no município de Monteiro Lobato-SP. Vale salientar, que este projeto inicial foi inclusive vencedor no XI concurso da Universidade Solidária-UNISOL naquele mesmo ano. O objetivo na época era proporcionar o acesso ao conhecimento técnico da atividade, possibilitando aos apicultores da Associação dos Pequenos Produtores Rurais-APPR de Monteiro Lobato a melhoria nas instalações da casa do mel, assistência técnica e o acompanhamento que envolveu conceitos técnicos e gerenciais para viabilizar a comercialização no mercado formal (BARRETO et al., 2012; PASIN; BARRETO, 2011; PASIN, 2007; PASIN; TERESO, 2008). Nos três primeiros anos desta caminhada, experiências das mais diversas foram acontecendo e proporcionaram o transbordamento das ações iniciais que primeiramente eram pautadas somente nas questões técnicas.

Com o andamento do projeto surgiram novas demandas e novos resultados. Isto sem mencionar o alcance aos próprios objetivos iniciais que eram o aumento da produção com qualidade e também a construção da casa do mel para uso coletivo onde, neste caso, o foco era a obtenção de um produto com qualidade e higiene que atendesse às determinações da vigilância sanitária, proporcionando assim a capacidade de se comercializar o produto mel no mercado formal.
Em função dos resultados alcançados pelo grupo de apicultores familiares de Monteiro Lobato, o projeto foi ampliado para mais 02 municípios do Vale do Paraíba-SP (Redenção da Serra e São Luiz do Paraitinga), inclusive com a participação de novos parceiros e apoiadores, que são as associações de apicultores de Redenção da Serra e São Luiz do Paraitinga, o poder público local e a iniciativa privada, neste caso representado pela empresa Fibria.

Atualmente o maior desafio dos grupos está na capacidade de se organizar para conquistar de maneira plena o acesso aos novos mercados institucionais (Programa de Aquisição de Alimentos-PAA e ao Programa Nacional de Alimentação Escolar - PNAE), pois estas novas oportunidades têm provocado o surgimento de novos empreendimentos associativos, que possibilitam a comercialização da produção ou parte dela.

Em contrapartida, neste novo cenário surge a necessidade de reestruturação interna dos empreendimentos, para atender às exigências destas novas formas de relações comerciais e principalmente surge a demanda para o desenvolvimento de arranjos socioprodutivos que possam estabelecer um novo modelo que permita aos grupos e empreendimentos coletivos do setor apícola alcançar as novas perspectivas de mercado.

Desta forma o presente artigo visa analisar o processo de desenvolvimento do arranjo socioprodutivo apícola no Vale do Paraíba-SP, sendo que o objeto de estudo se constitui no desenvolvimento e resultados das ações dos apicultores familiares dos municípios de Monteiro Lobato, Redenção da Serra e São Luiz do Paraitinga, juntamente com o Centro de Estudos Apícolas da Universidade de Taubaté, a parceria com o poder público local e o apoio da iniciativa privada.

O artigo inicia a discussão com uma breve exposição sobre o processo de formação das organizações socioprodutivas dos apicultores familiares a partir do ano de 2007. Além da seção introdutória, o artigo apresenta mais 04(quatro) seções: a segunda seção contém um breve recorte do universo de estudo; a terceira seção compreende uma síntese da caracterização da metodologia "Ciranda do Mel" junta-

\begin{tabular}{|l|l|l|l|l|}
\hline Agric. Fam. & Belém-PA & n. 10 & p. 31-38 & dez. 2014 \\
\hline
\end{tabular}


mente com os principais aspectos descritivos que envolvem as atividades de formação dos arranjos socioprodutivos e seus resultados; na quarta seção a análise se concentra na revisão bibliográfica envolvendo os aspectos relacionados aos desafios organizacionais que os apicultores familiares irão enfrentar para alcançar o mercado institucional e a quinta seção se encerra com as considerações finais.

\section{O UNIVERSO DE ESTUDO}

A área de abrangência do estudo compreende os municípios de Monteiro Lobato, Redenção da Serra e São Luiz do Paraitinga, onde $43,59 \%$ dos habitantes é da zona rural (Tabela 01). Estes municípios pertencem a região do Vale do Paraíba-SP que se localiza no cone leste paulista do estado de São Paulo e faz divisa com os estados de Minas Gerais e Rio de Janeiro.

Grupos Participantes:

- Apicultores Familiares e Grupos Familiares; - Associação de Pequenos Produtores Rurais de Monteiro Lobato-APPR (Núcleo Apicultura);

- Associação Socioeducativa de Produtores Rurais de Redenção da Serra- (Núcleo Apicultura);

-Associação de Apicultores de São Luiz do Paraitinga-APISTINGA;

- Cooperativa de Apicultores do Vale do Paraíba-COAPVALE.

Agentes:

- Professores, Pesquisadores e Estudantes;

- Centro de Estudo Apícola CEA/UNITAU;

Parceiros:

- Prefeitura Municipal de Monteiro Lobato;
Prefeitura Municipal de Redenção da Serra e Prefeitura Municipal de São Luiz do Paraitinga;

- Coordenadoria de Assistência Técnica Integral-CATI/SP;

- Secretaria da Agricultura do estado de São Paulo-SAA/SP;

- Serviço de Apoio a Micro e Pequena Empresas-SEBRAE/SP;

- FIBRIA - Papel e Celulose;

- Universidade Solidária - UNISOL (ABN/ AMRO BANK - SANTADER).

\section{O MÉTODO “CIRANDA DO MEL": SEUS AVANÇOS E SUAS CONQUISTAS}

A metodologia de ações participativas, denominada de "Ciranda do Mel" é fruto do resultado de diversas pesquisas e se fundamenta na atuação coletiva entre os participantes e o envolvimento dos familiares, onde durante a execução do programa são realizadas visitas mensais, treinamentos; discussão das ações; aplicação de tarefas e rodízio de acompanhamento dos resultados específicos nos apiários dos apicultores participantes (BARRETO et al 1994; SILVA, 2000; BARRETO et al 2004; SILVA; RICHETTO; SOUZA, 2005; BARRETO et al., 2006; PASIN, 2007; PASIN; TERESO, 2008; BARRETO et al., 2012, PASIN, 2011).

Juntamente com o ciclo mensal de visitas o programa prevê a criação do apiário-escola, onde todos os participantes são responsáveis pela condução e manejo deste apiário. Este processo permite que o aprendizado e o conhecimento sejam desenvolvidos de maneira prática e objetiva e, posteriormente, se consolide no próprio ambiente de trabalho do

Tabela 1. Os municípios da área de abrangência do programa

\begin{tabular}{l|r|l|l|l}
\hline \multirow{2}{*}{ Município } & \multicolumn{3}{|c|}{ População } & \multirow{2}{*}{ IDHM } \\
\cline { 2 - 4 } & Habitantes & Rural & Urbana & \\
\hline Monteiro Lobato & 4.381 & $56,40 \%$ & $43,60 \%$ & 0,710 \\
Redenção da Serra & 3.952 & $42,90 \%$ & $57,10 \%$ & 0,657 \\
São Luiz do Paraitinga & 10.721 & $40,50 \%$ & $59,50 \%$ & 0,697 \\
\hline \multicolumn{1}{c}{ Total } & 19.054 & $43,59 \%$ & $56,41 \%$ & \\
\hline
\end{tabular}

Fonte: IBGE (2012) 
apicultor que é o seu apiário. Além disso, o método prioriza o desenvolvimento do trabalho no grupo familiar, onde diversas ações culturais, orientações e capacitações profissionais são desenvolvidas com os familiares dos apicultores.

Desta forma entende-se que o desafio se consiste não somente em promover a construção coletiva e participativa em empreendimentos associativos, mas também proporcionar os aspectos intrínsecos que viabilizem a sustentabilidade destes empreendimentos (COLETIVA..., 2013)

Vale salientar que a "Ciranda do Mel" com o passar do tempo incorporou novas percepções e propósitos, que permitiram ampliar o horizonte dos apicultores e seus familiares, alcançando um nível de arranjo e organização socioprodutiva que demandou nesta caminhada inclusive a articulação e parceria com novos atores e agentes (tanto no setor público como no setor privado), viabilizando assim o alcance em outras áreas além da apicultura, como o desenvolvimento das artes, artesanato, culinária, cosméticos e produção de olerícolas.

De certa forma a caminhada para o desenvolvimento da apicultura familiar sustentável é constituída de 02(duas) etapas. A primeira etapa compreende o período de 2007 a 2010 onde se iniciam as atividades de formação do arranjo socioprodutivo, envolvendo neste caso somente os apicultores familiares da Associação dos Pequenos produtores Rurais-APPR do município de Monteiro Lobato (Barreto et al 2012).

Nesta primeira etapa (período 2007 a 2010) os principais resultados alcançados foram:

- Participação de mais de 40 pessoas diretamente envolvidas (apicultores e familiares) e mais de 120 pessoas envolvidas indiretamente;

- Aumento da participação da apicultura na renda familiar (de $23,6 \%$ para $25,7 \%$ );

- Aumento no número de colmeia no apiário (de 20,8 para 22,8 colmeias/apiário);

- Elevação da produtividade de mel por colmeia (de 11,5 quilos para 19,4 quilos de mel/ colmeia/safra);
- Implantação da infraestrutura coletiva (completa) para o beneficiamento do mel;

- Criação e desenvolvimento do Grupo Jovens Apicultores;

- Ampliação da consciência ambiental (preservação da mata com diversas espécies de plantas que são utilizadas na apicultura) e das competências empreendedoras entre os apicultores e seus familiares;

- Maior envolvimento dos apicultores e seus familiares em relação à diversificação dos negócios relacionados à apicultura na região (culinária; cosméticos derivados do mel e Agroturismo);

- Criação do Grupo teatral (constituídas por filhos de apicultores);

- Elaboração da rota turística "Trilha do Mel" (atividade relacionada ao Agro Turismo);

- Retorno de apicultores aos bancos escolares e a melhora do desempenho escolar (neste caso são alguns apicultores já adultos que retomam seus estudos e filhos de apicultores que passam a se dedicar mais aos estudos);

- Criação do grupo de artesãs (são apicultoras, mulheres pertencentes às famílias dos apicultores e também mulheres moradoras das comunidades próximas);

- Maior articulação com o poder público local (permitindo a conquista de apoio efetivo em questões técnicas, infraestrutura e melhoria nas condições de acesso e estradas dos bairros rurais).

Já a segunda etapa (período de 2010 a 2013) apresenta o interstício que tem como característica principal a expansão das atividades para os municípios de Redenção da Serra e São Luiz do Paraitinga, onde a presença de novos parceiros viabiliza a inclusão dos apicultores familiares dos respectivos municípios. Nesta etapa a abrangência geográfica se expande para 03 (três) municípios, e os principais resultados alcançados foram:

- Inclusão e participação de mais de 150 pessoas diretamente envolvidas (apicultores e familiares) e mais de 450 pessoas envolvidas indiretamente pertencentes aos municípios de Redenção da Serra e São Luiz do Paraitinga;

- Instalação do Centro Comunitário no bairro rural da Pedra Branca no município de Monteiro Lobato-SP;

\begin{tabular}{|c|c|c|c|c|}
\hline Agric. Fam. & Belém-PA & n. 10 & p. 31-38 & dez. 2014 \\
\hline
\end{tabular}


- Obtenção da certificação do Serviço de Inspeção Municipal-SIM para os apicultores da APPR do município de Monteiro Lobato;

- Criação da Cooperativa de Apicultores do Vale do Paraíba COAPVALE com sede em Redenção da Serra;

- Venda institucional do produto mel (Atacado-balde e Fracionado-sachet) para o programa público de aquisição de alimentos para merenda escolar;

- Realização do I Seminário Estadual Paulista de Apicultura e Meliponicultura-FAAMESP no Centro de Estudos Apícolas-CEA/UNITAU;

- Assinatura do convênio entre a Secretaria da Agricultura do estado de São Paulo para fomentar o Arranjo Produtivo da Apicultura na região, onde a governança será de responsabilidade do Centro de Estudos Apícolas-CEA/UNITAU.

\section{OS DESAFIOS DO MERCADO INSTI- TUCIONAL E OS NOVOS CAMINHOS A SE- REM TRILHADOS.}

A atividade empreendedora, seja no meio urbano ou rural, nas últimas décadas tem ocupado papel de destaque na sociedade, observando que o crescimento e o desenvolvimento econômico, social e ambiental das nações ocorreram graças ao comportamento empreendedor, tanto nos países desenvolvidos como nos países em desenvolvimento (TIMMONS, 1989; MURPHY; LIAO; WELSCH, 2006; CORNELIUS; LANDSTROM; PERSSON, 2006; GREGOIRE et al., 2006).

Como agente socializante, a universidade, inspirada em modelo teórico semelhante ao hélice-tríplice, porém com proatividade e detentora da responsabilidade de governança, deve propor, além de pesquisa tecnológica, ações extensionistas que promovam o desenvolvimento sustentável (AUDY; FERREIRA, 2006; CLARK, 2006; DEGEN, 2008; ETZKOWITZ; LEYDESDORFF, 1996; ETZKOWITZ, 2005; TERRA et al., 2006; MELLO, 2004).

Para Degen (2010) a combinação entre ambiente inovador, oportunidade e cultura empreendedora proporciona o alicerce para o desenvolvimento sustentável. Portan- to, estimular a organização de arranjo socioprodutivo e promover o comportamento empreendedor entre os apicultores familiares é extremamente fundamental, pois o setor apícola e a própria agricultura familiar na atualidade têm revelado um papel importante para o desenvolvimento rural sustentável, bem como a própria garantia da produção de alimentos para o abastecimento agroalimentar da população (BECKER; ANJOS, 2010; ABRAMOWAY, 1992; WILKINSON, 2008).

De acordo com Silva e Silva (2011) o estímulo à produção rural familiar pode ser considerado uma opção estratégica para o desenvolvimento endógeno sustentável nos diferentes contextos territoriais, sendo que muitas vezes, os próprios aspectos tradicionais e locais da pequena produção se transformam em valores de mercado, que quando bem trabalhados no território, podem proporcionar $\mathrm{o}$ alcance de maior renda, pelo produtor, promovendo a dinâmica das economias locais.

Para Wilkinson (2008), temas presentes e interdependentes dominam os esforços de construir ou consolidar os novos mercados, a partir da agricultura familiar. Neste caso, o que estes novos mercados têm em comum é a necessidade de definir, negociar e operacionalizar um novo processo de organização socioprodutiva, sendo que na sua abrangência, isto implica na formalização de ações coordenadas, baseadas no reconhecimento da especificidade da região e da própria agricultura familiar.

No caso específico da apicultura familiar pode-se afirmar que ações desta natureza requerem inovações institucionais de cooperação e parcerias entre o agente público e privado. Vale salientar, que a caracterização da apicultura familiar como estratégia de desenvolvimento local, exige também uma reinvenção de tradições, onde a adoção de novas práticas de produção e gestão se faz necessária para permitir o acesso às novas possibilidades de atuação em mercados emergentes.

Desta forma, entende-se que o processo que vem sendo desenvolvido na atividade da apicultura nos municípios de Monteiro Lobato, Redenção da Serra e São Luiz do Paraitinga, se aproxima muito das caraterísticas 
que devem estar presentes para a construção e desenvolvimento de ações inovadoras que permitam potencializar a capacidade de se aproveitar as oportunidades e estabelecer uma nova relação entre os agentes presentes na região, e principalmente viabilizar condições concretas para que se possa promover de fato a inserção dos novos empreendedores apícolas no mercado institucional, proporcionado assim a geração de trabalho e renda entre os apicultores familiares da região.

Vale lembrar que no início da caminhada, a atividade produtiva era desenvolvida, muitas vezes, em condições inadequadas que comprometia não somente a produtividade, como também, em função das instalações inapropriadas, a higiene e qualidade do produto final.

Entretanto com o passar do tempo, e com o próprio avanço das ações e programas que foram sendo desenvolvidos, primeiramente na apicultura do município de Monteiro Lobato e posteriormente nos municípios de Redenção da Serra e São Luiz do Paraitinga, pode-se afirmar que os resultados positivos alcançados permitiram aos apicultores desenvolverem condições reais para obtenção de um produto final de plena qualidade, que atende inclusive as exigências da vigilância sanitária da região.

Em relação à aquisição de alimentos mediante ao Programa de Aquisição de Alimentos-PAA e ao Programa Nacional de Alimentação Escolar - PNAE, pode-se afirmar que esta oportunidade é um instrumento de grande valia para estimular o incremento da produção rural, pois graças a este sistema de aquisição e abastecimento dos órgãos públicos, é possível viabilizar que os agricultores tenham acesso a mercados institucionais fora do contexto da lógica concorrencial do mercado capitalista (SABOURIN, 2009). Entretanto, Silva e Silva (2011) alertam para os problemas a serem enfrentados no que diz respeito à consolidação do estímulo à produção familiar agrícola, que vise o alcance e oferta ao mercado institucional, pois segundo pesquisa realizada, em 2010 pelo Centro Colaborador em Alimentação e Nutrição Escolar da Universidade Federal de Ouro Preto, entre
478 compradores (gestores públicos de diversos municípios de Minas Gerais responsáveis pela aquisição de alimentos da agricultura familiar) $45 \%$ apresentaram como principal entrave a razão do empreendimento rural familiar não estar formalmente organizado, o que acaba promovendo dificuldade para o acesso ao mercado institucional.

Portanto, estas novas possibilidades revelam que o estímulo à exploração de novos empreendimentos e o aumento da produção rural familiar, que possibilite o incremento na renda da família, devem ser considerados uma ação estratégica fundamental para o desenvolvimento sustentável na região. De acordo com Haddad (2009) o processo de desenvolvimento endógeno de uma região depende não só do seu crescimento econômico, mas também da sua capacidade de articulação e implementação de políticas, programas e projetos concebidos para atender diversos setores da sociedade, priorizando principalmente a participação dos atores locais como protagonistas no processo de formação dos arranjos socioprodutivos.

No caso da apicultura familiar nos municípios dos municípios de Monteiro Lobato, Redenção da Serra e São Luiz do Paraitinga pode-se afirmar que para atender as demandas relacionadas à aquisição de alimentos, via mercado institucional (PAA e PNAE), o arranjo socioprodutivo ainda se encontra em processo inicial.

No entanto, vale ressaltar que a Cooperativa de Apicultores do Vale do Paraíba-Coapvale, criada em 2012, é também resultado dos desdobramentos das ações inovadoras e empreendedoras que foram sendo desenvolvidas nos últimos anos entre apicultores familiares que participaram dos programas e da metodologia "Cirandas do Mel". Esta cooperativa tem como um de seus objetivos principais, viabilizar o processo de comercialização no mercado institucional, mediante a conquista da certificação do serviço de inspeção da vigilância sanitária e ajuste da própria capacidade de oferta do produto a este canal de comercialização, fato este que ainda não ocorreu de maneira plena.

Porém pode-se dizer que o processo

\begin{tabular}{|l|l|l|l|l|}
\hline Agric. Fam. & Belém-PA & n. 10 & p. 31-38 & dez. 2014 \\
\hline
\end{tabular}


evolutivo desta caminhada obteve significativas conquistas, uma vez que a condição de manejo e extração do mel em situações inadequadas, onde muitas vezes era realizada na própria cozinha da residência do apicultor hoje é "coisa do passado", pois as atividades de extração e beneficiamento do mel são realizadas em instalações que atendem plenamente as exigências da vigilância sanitária, o que certamente viabiliza a prática da comercialização no mercado formal e institucional.

\section{CONSIDERAÇÕES FINAIS}

A apicultura familiar, presente nos municípios integrantes, apresenta-se como uma atividade de exploração com possibilidades de crescimento, onde o aumento da produção de mel deverá ocorrer juntamente com o aumento da participação do apicultor no mercado institucional.

Este crescimento de produção condicionado à inserção do apicultor no mercado institucional estabelece um novo modelo de desenvolvimento para a apicultura familiar, onde os agentes parceiros, seja público ou privado, têm demonstrado a sua importância e papel no processo de formação do arranjo socioprodutivo da apicultura familiar.

Neste caso, os programas de fomento para o setor deverão se basear em novos modelos que possuam como principal eixo condutor o entendimento do papel da apicultura na região, sua importância econômica como atividade geradora de renda e a própria potencialidade de crescimento, através da conquista do mercado formal e institucional existente inclusive na região.

É fundamental que o apicultor familiar, as associações e parceiros (público e privado) de maneira organizada estabeleçam um modelo arranjo socioprodutivo de desenvolvimento sustentável para a apicultura regional, amparado na alta produção de mel com qualidade, visando principalmente aproveitar a oportunidade de abastecer o mercado institucional da região.

Desta forma entende-se que metodologias de ações participativas, como é o caso da "Ciranda do Mel", devem ser replicadas e difundidas em diversas localidades que apresentem potencial para o desenvolvimento da apicultura e que tenha como base o trabalho da mão de obra familiar, pois o sucesso da metodologia "Ciranda do Mel" está fundamentada na capacidade de promover a participação plena do empreendedor apícola familiar no processo e principalmente proporcionar o empoderamento a este apicultor.

\section{REFERÊNCIAS}

BARRETO, L.M.R.C. et al. . Ciranda do mel: o desenvolvimento socioambiental da apicultura familiar no Vale do Paraíba-SP. Revista Ciência em Extensão. v. 8, n. 3, p.284-289, 2012.

BARRETO, L.M.R.C., et al. Resultados preliminares da difusão de abelhas rainhas do Vale do Paraíba (projeto DIFAR, Manejo e Introdução). In: FORO EXPO-COMERCIAL INTERNACIONAL DE APICULTURA, 1994, Buenos Aires-AR. Anais...maio 1994, p.69-72.

\section{BARRETO, L.M.R.C. et al. Higienização e sa-} nitização na produção apícola. Taubaté, SP: Cabral Editora Livraria Universitária, 2006.

\section{COLETIVA II: Sistematizações sobre a prá-} tica autogestionária. Campinas: Instituto de Economia, Unicamp, 2013, 298 p.

PASIN, L.V.E; TERESO, M.J.A. Análise da infraestrutura existente em unidades de produção agrícola para o processamento de mel na região do Vale do Paraíba-SP.Ciência e Agro tecnologia. Lavras, MG. v.32, n.2, p. 510-516, mar/abr, 2008.

PASIN, L.V.E. A Organização da produção e comercialização do produto mel: a apicultura no Vale do Paraíba-SP. Tese (Doutorado) - Faculdade de Engenharia Agrícola. Universidade Estadual de Campinas. Campinas-SP, 2007. 
PASIN, L.V.E.; BARRETO, L.M.R.C. Desenvolvimento socioambiental da apicultura familiar no município de Monteiro Lobato. In: CONGRESSO BRASILEIRO DE EXTENSÃO UNIVERSITÁRIA, 5. Anais... Porto Alegre-RS, 2011.

PASIN, L.V.E. Sistemas sócio produtivos e o desenvolvimento regional: uma reflexão para produção de pólen no Brasil. Revista Magistra. Cruz das Almas, v.23, ed. esp., p. 38-45, 2011.

SILVA, W.P. Manual de comercialização apícola. Maceió-AL: SEBRAE, 2000.

SILVA, M.G. da; SILVA, S.P. Para além do acesso: uma análise da relação entre mercados institucionais e empreendimentos de economia solidária no meio rural. Mercado de trabalho: conjuntura e análise. Brasília, v. 16, nov, 2011.

SILVA, R.J.; RICHETTO, K.C.S.; SOUZA, M.M.S. Padrões de identidade e qualidade dos méis comercializados na Rodovia Presidente Dutra. In: ENCONTRO DE INICIAÇÃO CIENTÍFICA, 10. Anais...Taubaté: Universidade de Taubaté, 2005. 